\title{
Study on call admission control schemes in 3GPP LTE network
}

\author{
Maniru Malami Umar a,1, , Aminu Mohammed a,2, Abdulhakeem Abdulazeez a,3, Solomon \\ Ordeun Yese ${ }^{a, 4}$ \\ a Department of Mathematics, Usmanu Danfodiyo University, Sokoto, Nigeria \\ 1 manirutambuwal@gmail.com \\ * corresponding author
}

\section{ARTICLE INFO}

Article history

Received September 27, 2020

Revised October 15, 2020

Accepted November 20, 2020

Keywords

Throughput

Call Admission Control

LTE

Radio Resource Management Call Blocking Probability (CBP) Call Dropping Probability (CDP)

\begin{abstract}
Call admission control (CAC) is a process of accepting new calls or handoff calls in a network while regulating the Quality of Service (QoS) of existing or active calls without degrading any call drop. CAC is an RRM technique and directly impacts QoS for individual connection and the overall system efficiency. This paper presents a short review of some existing CAC schemes proposed for the $3 \mathrm{GPP}$ LTE network. The review aims to guide researchers to know what CAC is, how it operates, and its benefits to the overall system performance. The schemes reviewed can be classified based on the aim they were proposed, such as guaranteeing QoS of calls, increasing the utilization of available network resources, reducing call blocking, and call dropping probabilities. The paper further summarised all reviewed schemes by highlighting each scheme's operations, strengths, and weaknesses.
\end{abstract}

This is an open access article under the CC-BY-SA license.

\section{Introduction}

Quality of Service (QoS) provisioning is one of the primary objectives of any wireless broadband technology. Long Term Evolution (LTE) is one of the wireless broadband technologies focused on QoS provisioning. A user connected to the network always expects a better QoS experience. Mobile operators have always worked tirelessly to improve the QoS of their users [1]. LTE employs several Radio Resource Management (RRM) techniques to ensure that the QoS users are satisfied to a certain level of satisfaction. An efficient RRM technique that will handle the network resources efficiently is required because network resources are, in most cases, scarce [2]. Specifically, an efficient call admission control (CAC) scheme which regulates resources for new call requests or ongoing calls is needed. Call admission control is the process of accepting a new call or a handoff call request into the network while maintaining the quality of service (QoS) of admitted or ongoing calls [2]. Call requests are classified into two: new call and handoff call requests. A new call is a call request that is requesting a new connection into the network. In contrast, a handoff call is an ongoing or already connected call that needs to be transferred from one cell to another without compromising service quality [1].

This paper aims to give an over of the 3GPP LTE networks, i.e., what it is, how it operates, its architecture, and the primary objectives. The paper will further examine an RRM technique that 
helps regulates the QoS of existing and new calls in a network. Several existing CAC schemes have been reviewed in this paper by highlighting each of the schemes' operations, strengths, and weaknesses. The rest of this paper is organized as follows: Section 2 presents an overview of the 3GPP LTE network, call admission control is presented in section 3 of this paper. Call admission control in LTE is presented in section 4 , and section 5 concludes the paper.

\section{Method}

\subsection{GPP Long Term Evolution (LTE)}

Long Term Evolution (LTE) is one of the wireless broadband technologies that focused on QoS provision for different users. Long Term Evolution (LTE) is an evolving wireless standard developed by the 3rd Generation Partnership Project (3GPP), along with 3GPP HSPA+, 3GPP EDGE Evolution, and Mobile WiMAX (IEEE 802.16e), opens the road to 4G technologies. The LTE standard is focused on delivering high data rates for bandwidth-demanding applications and improving flexibility and spectral efficiency, thus constituting an attractive solution for both users and mobile operators. LTE network was designed to deliver a peak data rate of $100 \mathrm{Mbps}$ in the downlink and 50Mbps in the uplink. This requirement was exceeded in the eventual system, which delivers peak data rates of $300 \mathrm{Mbps}$ and $75 \mathrm{Mbps}$ for the downlink and uplink, respectively [3].

The LTE architecture is also known as Evolved Packet System (EPS), comprises two main components, which are: Evolved Radio Access Network (E-UTRAN) and Evolved Packet Core (EPC) [4]. The E-UTRAN consists of a network of enhanced base stations referred to evolved NodeB (eNBs), whose primary function is to manage the available radio resources and mobility in the cell to optimize the communication among all User Equipment (UEs). On the other hand, the EPC is the core network that controls the activities of the user equipment (UEs). It comprises of Mobility Management Entity (MME), Serving Gateway (S-GW), Home Subscriber System (HSS), and Packet Data Network Gateway (P-GW). The MME controls the mobile's high-level operation by sending it signaling messages about issues such as security and management of data streams that are unrelated to radio communication. On the other hand, HSS is a component that contains subscription data of the UE. It stores user authentication data and subscription status. S-GW handles the data and packet routing within the LTE, while P-GW handles data and packets routing towards non-3GPP data networks [5]. The system architecture of LTE is shown in Fig. 1.

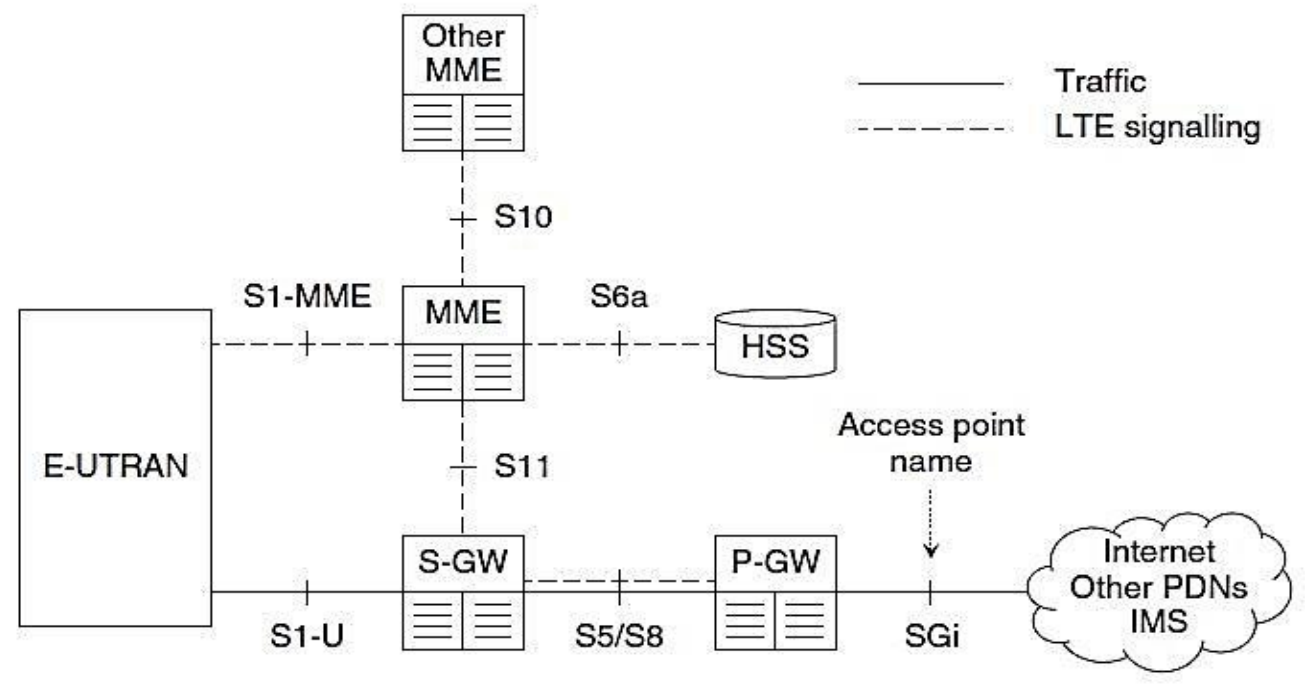

Fig. 1. LTE Architecture [5] 
The LTE radio interface supports three multiple access techniques, which are Orthogonal Frequency Division Multiple Access (OFDMA), Multiple Inputs Multiple Outputs (MIMO), and Single Carrier Frequency Division Multiple Access (SC-FDMA) techniques [6]. OFDMA is a multiple access technique used at the downlink channel in the LTE system, supporting high Quality of Service (QoS) to the accessing points. On the other hand, MIMO uses multiple transmitters and receivers to transfer more user data at the same time. The MIMO supports high coverage, high data rate and better robustness, low bit error rate, and better spectral efficiency. It is also used as a downlink channel. SC-FDMA is a multiple access technique that is used in the uplink channel of the LTE system.

LTE's fundamental objective is to guarantee quality of service (QoS) requirements and minimize network congestion, thereby utilizing the available network resources [7]. It can be achieved through the radio resource management (RRM) techniques. Wireless networks employ radio resource management techniques to improve the utilization of radio resources. Radio resources are utilized using various schemes that can are categorized into three major groups [8]. The first group represents frequency or time resource allocation schemes, including channel allocation, scheduling, transmission rate control, and bandwidth reservation schemes. The second group represents power allocation and control schemes, including the terminals and base stations' transmitter power. The third group represents access port connection schemes, including call admission control, base station assignment, handoff control algorithms, and call admission control.

\subsection{Call Admision Control (CAC)}

Call admission control (CAC) is a process of accepting new calls or handoff calls in a network while regulating the QoS of existing or active calls without degrading any call drop [7]. CAC is an RRM technique and directly impacts QoS for individual connection and the overall system efficiency [9]. Call admission control is located at layer three, i.e., network layer in the evolved Node B (eNB), and used for both new user and handoff users [10]. Call requests usually are classified as New Call (NC) and Handoff Call (HC). NC is a type of call requesting a new connection or requesting to be connected to the network, while HC is an ongoing or active call that needs to be transferred from one cell to another and still maintain its connection.

The primary objective of CAC is to ensure efficient resource allocation and to monitor the resource utilization in the high volume of traffic. CAC determines the condition for accepting or rejecting an $\mathrm{NC}$ or $\mathrm{HC}$ into the network based on pre-defined criteria such as availability of network resources, network channel condition, and others. To guarantee the QoS parameters without affecting the existing calls [11]. CAC process is always performed when a UE starts communication with the eNodeB either through a new call or a handoff call, or a new service request by the UE. When the UE wants to establish a connection with the eNodeB, it sends a request for resource allocation, admission control at eNodeB handles the request. For RT call requests, if connection causes excessive interference to the system, the request will be denied. Otherwise, resources will be allocated for that connection. For the NRT connection request, the packets' optimum scheduling must be determined after the admission of the call [12].

CAC schemes usually are designed for a specific purpose, such as guaranteeing the QoS of calls, increasing the throughput of the system, increasing the utilization of the available network resources, thereby reducing the wastage of the resources. Some of the schemes are meant for reducing the call blocking probability (CBP), call dropping probability (CDP), and others. The 3GPP standard does not define any standard for call admission control. It has been left open for vendors and network operators to decide how the CAC schemes are developed.

Basic Call Admission Control (BCAC) is a static call admission control scheme [2]. The decision for the acceptance and rejection of a call request depends only on network resource availability. Call requests are only admitted into the network when the requested resources are less than or equal to the available network resources; otherwise, the call request is rejected. Therefore, in the BCAC scheme, the admission criteria always depend on the availability of network resources. Fig. 2 describes the operation of the BCAC scheme. 


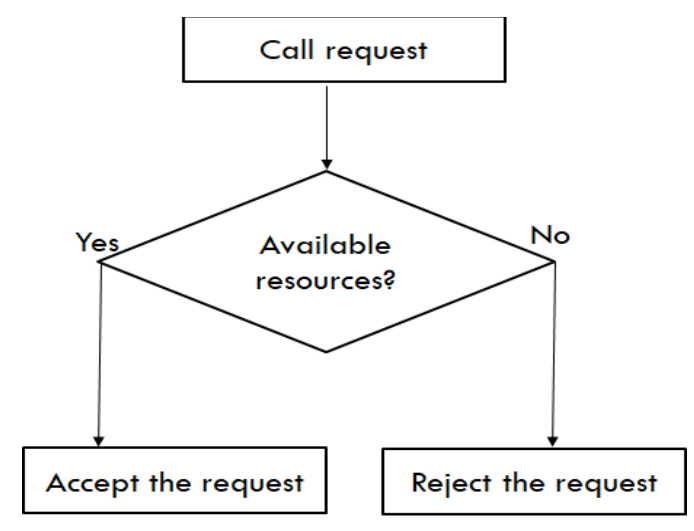

Fig. 2. BCAC Description

The multi-service call admission control (MSCAC) was proposed for 3G/4G networks to improve the BCAC scheme. MSCAC supports two types of services; RT and NRT, where RT is for conversational and streaming calls, while NRT is for BE. The scheme divides radio resources into two parts, one part for the RT calls and the other part for NRT calls.

A CAC scheme's design depends on some parameters such as availability of resources, quality of network parameters, quality policies, call prioritization, mobility management, and optimization methodologies, and others [8].

\section{Results and Discussion}

Researchers want to dive into call admission control, as an area of research needs first to overview the existing CAC schemes proposed for particular network technology, in this case, LTE. Several CAC schemes have been proposed with different aims and objectives, such as guaranteeing QoS, reducing call dropping probability (CBP), and call blocking probability (CD).

This section presents some of the CAC schemes developed for the LTE network. The description, strengths, and weaknesses of each of the schemes are also highlighted. Finally, all the schemes have been summarised in a tabular form of simplicity.

In [13], the authors presented a novel admission control scheme for multiclass services in LTE systems to reduce the CBP and guarantee the users' QoS. The scheme combines complete sharing (CS), virtual partitioning (VP), and service degradation strategies. It groups users into three groups: group 1 are services whose resources can be preempted, group 2 are services whose resources cannot be preempted, and group 3 are services that can preempt resources from group 1 . The scheme admits a new call request of service group 1 if the available bandwidth in group 1 is greater than or equal to the requested bandwidth; otherwise, the call is rejected. Similarly, it accepts a new call of service group 2 if the available bandwidth in groups 2 and 3 is greater than or equal to the requested bandwidth. Otherwise, bandwidth is degraded from admitted calls in the group. If the degraded bandwidth is enough to admit the new call, the call is accepted; otherwise, the call is rejected.

Furthermore, the scheme accepts a new call of service group 3 if the available bandwidth in service groups 2 and 3 is greater than the requested bandwidth; otherwise, the call is rejected. The scheme reduces the CBP of users and also guarantee the QoS of some service types. However, the QoS of lower priority users because they are degraded whenever resources are not sufficient. It also increases the CDP of these users.

The study in [14] proposed a resource-estimated CAC scheme to guarantee QoS for different traffic types. The scheme estimates the number of required Physical resource Blocks (PRBs) based on the service type, RT or NRT, and current Modulation and Coding Scheme (MCS) level of the user. It determines the minimum data rate required for each service type at the request time. The scheme accepts handoff requests with the lowest MCS level while rejects requests with the highest MCS level. It similarly accepts new calls with the lowest MCS level and rejects 
these with the highest MCS level. The scheme improves QoS and also increases resource utilization. However, the scheme increases handoff dropping probability because the MCS level for handoff calls at request time is higher than the new calls.

In [15], a Delay Aware Call Admission Control (DACAC) scheme guarantees QoS for different service call requests using a dynamic measurement strategy to determine Packet delay and PRB utilization of each service type. The scheme models use two thresholds: Threshold 1 (TH1) and threshold 2 (TH2) for PRB utilization. The scheme accepts a call when its arrival time is equal or less than TH1; otherwise, the call is rejected if the service arrival time is equal to or greater than TH2. It also accepts a call when the service arrival time is more excellent than TH1 but less than TH2. It accepts handoff calls but rejects a new call in the presence of congestion. The scheme guarantees QoS for different service types but increases the new call blocking probability when the network is congested.

Downlink CAC with look-ahead calls to handle advance resource reservation was presented in [16]. The scheme deals with three admission requests: new immediate call, handoff call, and advance call. It accepts a new immediate call when the new call's sum and that of aggregated active new calls is less than the new call capacity threshold; otherwise, the call is rejected. Similarly, the scheme accepts a handoff call if the sum of the handoff call and that of the aggregated active handoff calls is below the handoff capacity threshold; else, the call is queued. The queued calls are treated based on FIFO discipline if there is more than one call in the queue.

Furthermore, it accepts an advance call when the sum of advance call and the aggregated active advance calls are less than the advance call threshold; otherwise, the call is rejected. The scheme utilizes resources efficiently when traffic is low, but resources are underutilized when the traffic is high due to the advance call resource reservation. The scheme also increases blocking probability for new immediate calls and handoff calls due to higher priority given to advance calls.

In [17], a two-stage call admission control scheme guarantees the QoS of both new and active calls and improves system resource utilization. The scheme also incorporated a Packet scheduler (PS), which determines a call request's priority. It employs a dynamic threshold SINR for admitting new calls into the system. The scheme operates in two stages; At stage one, a new call request is accepted if the gain link between the transmitter and the receiver is more than a minimum threshold value set. Similarly, at stage two, the scheme tries to guarantee a minimum data rate for Guaranteed Bit Rate (GBR) requests. It considers the capacity of the established link and then quantifies it in terms of reserved system resources. It accepts a request when the requested resources are less than the available and reserved system resources; otherwise, the request is rejected. The scheme further employs a PS that determines each request's priority before the distribution of system resource commences. It gives higher priority to GBR and then assigns the lowest priority to NBGR. The scheme reduces call blocking and dropping probability for GBR requests, guarantees QoS of admitted calls, and increases overall system capacity. However, the scheme increases call blocking and call dropping probability of NGBR requests due to higher priority given to GBR requests.

In [11], the authors proposed an adaptive call admission control scheme to reduce the handoff dropping probability of handoff calls. The scheme employs a resource block (RB) reservation strategy, which gives higher priority to handoff calls and reserves a certain amount of resources. It employs a load balancing mechanism that adjusts the number of resources to be reserved for hand off calls. The scheme accepts a new call request when the requested resources are less than or equal to the available resources; otherwise, the call is rejected. It admits a handoff call when the requested resources are less than or equal to the available and reserved resources for handoff calls; otherwise, the call is queued into a waiting queue. It served the queued calls based on their latency. The scheme reduces the handoff dropping probability of handoff calls because they are given higher priority but increases the call blocking probability of new call requests. It also reduces the utilization of network resources because of the reservation strategy employed by the scheme. 
The study in [18] proposed a Utility-Based Scheduling and Called Admission Control (UBSCAC) scheme to reduce CBP and CDP of RT and NRT users. The scheme estimates channel conditions based on received signal strength (RSS) to determine good and bad channels. The scheme classifies requests into NC and HC and categorizes RT requests as VoIP and Video. It checks for the bandwidth requirement of a VoIP request. If the requested bandwidth is less than the total available bandwidth, it can be reserved based on the base station's traffic density, and the request admitted. Otherwise, if the requested bandwidth is more than the total available bandwidth, then bandwidth reservation is made using resources from bad channels. The scheme admits a Video request if the requested bandwidth is less than or equal to the remaining available bandwidth, else the request is rejected. It also admits Video requests with the Lowest Tolerance of Latency (TOL) when there are multiple requests at a time. The scheme reduces CDP for HC but increases CBP for NC due to higher priority given to HC. Also, users with bad channels are starved due to the degradation strategy used by the scheme.

In [19], a Hybrid Adaptive Call Admission Control (HCAC) scheme reduces the handoff blocking probability. The HCAC scheme uses a resource block strategy to allocate resources based on either a new call or a handoff call. It checks for the maximum number of Resource Blocks (RBmax), the number of required RBs (RBreq), the minimum number of RBs (RBmin), and the maximum tolerable delay (Dmax) on the arrival of a call. The scheme checks the RBreq and RBmin for new calls and handoff calls, respectively. It checks if the latency is less than the maximum tolerable delay, then the call with minimum latency is checked, and the resource block strategy is used. The scheme employs an expiration delay to service classes when resources are not available. It reduces handoff dropping probability but increases new call blocking probability under heavy traffic.

An efficient channel state-based call admission control for non-real-time traffic in LTE networks was proposed in [20] to guarantee the QoS and reduce users' CBP. The scheme classifies call requests into the new call (NC) and handoff call (HC) and further categorizes each class as RT and BE traffics. It then estimates the channel condition based on the received signal strength (RSS) value. The scheme accepts a new call request if the requested bandwidth is less than or equal to the available bandwidth and the channel condition if the call is good. Otherwise, bandwidth is reserved based on the traffic density of the base station. It further degrades admitted calls with bad channel conditions when a new call request arrives, and there are no sufficient resources to admit the call. If the degraded bandwidth is not enough to admit the new call request, the call is rejected. The scheme guarantees QoS and also reduces the CBP of calls with good channel conditions. However, the scheme increases the CBP and CDP of calls with bad channel conditions.

The [21] proposed a call admission control scheme for the machine to machine (M2M) communication to reduce the delay experienced by calls before being admitted into the LTE system. The scheme manages machine type communication (MTC) devices using a group-based strategy. It groups a set of MTC devices with the same QoS requirements in the same cluster. The scheme accepts a call request first by checking if a cluster exists that satisfies the call's QoS requirements. If there exists a satisfying cluster, then it checks if there are available slots in the cluster. If there are available slots in the cluster, then the call is admitted; else, the scheme checks if the request satisfies the sufficient conditions for creating a new cluster. The scheme further checks if the delay constraints for all the clusters are satisfied, then a new cluster is created, and the request is grouped as part of the cluster. Otherwise, if the conditions for creating a new cluster are not satisfied and the delay constraints are not met, the call request is rejected. The scheme ensures that all clusters satisfy their delay constraints by reducing the delay experienced by call requests. However, it increases the blocking probability of a call since all delay constraints must be satisfied before a call can be admitted.

In [22], an admission control scheme for video telephony services in wireless networks reduces CBP and increases resource utilization of network resources. The scheme was developed to support two GBR services; conversational voice calls and conversation video calls. It gives higher priority to conversational voice call requests. The scheme accepts a voice call 
request if the requested resources are less than or equal to the reserved and allocated resources to voice calls; otherwise, the request is blocked. It also accepts a voice call request by applying a degradation mechanism of the already admitted video calls when there are insufficient resources to admit the new voice call request, leading to the degradation of the quality of admitted video calls. Furthermore, the scheme accepts a video call request if the requested resources are less than or equal to the system's available resources; else, the request is rejected. The scheme reduces CBP and CDP of voice calls because they are given higher priority but lower priority calls, i.e., video calls experience increased CBP and CDP.

Researchers in [23] presented a QoS based call admission control and resource allocation scheme for LTE femtocell networks to prevent resource wastage and prevent call quality degradation for voice and data calls. The scheme monitors all calls through the home eNodeB gateway (HeNBGW) in a real time manner, and the mean opinion score (MOS) is always computed. The scheme triggered a CAC and dynamic resource allocation mechanisms periodically. It checks if the average MOS of a call is above the $95 \%$ confidence interval range. If it is, then the call is categorized as a type with a home area network (HAN) problem; otherwise, a new MOS is computed for the call. It accepts a new call if the average MOS is less than 3.8; otherwise, the call is rejected. The scheme maintains the QoS of voice calls due to the higher priority given to them. However, the QoS of lower priority calls, i.e., data calls, is not maintained because they are given lower priority.

In [24], the authors proposed a call admission control scheme for LTE femtocell networks to support multimedia services with diverse traffic classes and different bandwidth requirements. The scheme operates in two stages: The subscriber authentication stage and the admission control stage. Upon arrival of an E-UTRAN Radio Access Bearer (E-RAB) request, the scheme checks if the threshold based on subscriber authentication is not exceeded and then check if there are available PRBs in the system. The request is accepted if the conditions are satisfied; otherwise, the request is queued. The scheme accepts any queued request if it satisfies the predefined admission criteria and then leaves the queue's remaining requests. It rejects the remaining queued request when they reach their queue timeout. The scheme reduces CBP for each class of traffic and also increases resource utilization. However, it increases CDP when the queued requests reach the queue timeout.

Reasonable, intelligent admission control to provide fair resource allocation and guarantee maximum resource utilization for different service types was proposed [25]. The scheme combines complete sharing (CS) and virtual partitioning (VP) resource allocation techniques. It uses CS for multiclass users to share available network resources. The scheme further uses VP to differentiate among multi-service users when the network resources are scarce. It classifies call requests and categorizes the requests as GBR and MBR based on their service types. The scheme give higher priority to GBR. It accepts a higher priority request by applying a step-wise degradation approach that degrades resources allocated to lower priority bearers when there are insufficient resources to admit the request. It admits lower priority calls when there are enough resources; otherwise, the call re rejected. The scheme reduces call blocking probability for higher priority calls and guarantees fair resource sharing among service types. However, the scheme increases call blocking and call dropping probability for lower priority calls.

In [26], a fuzzy approach calls admission control to improve network resource utilization, reduce CBP and CDP, and ensures that the QoS of both new and ongoing calls is met. The scheme only deals with data services, i.e., lower priority calls. The scheme performs a channel aggregation when a new or handoff call request arrives. It assigns a channel or combination of channels to a call request to meet the expected throughput required to service the call request. The scheme directly assigns one or more channels to a request that is admitted. It queued call requests that are not admitted and then performed a combination of channels to service the request; otherwise, the request is blocked or dropped after four trials of the channel combination. The scheme reduces call blocking and dropping probability for data services that 
have lower priority. It also ensures QoS provisioning for both new and handoff calls of data services. However, the scheme increases CBP and CDP of higher priority calls.

The study in [27] proposed a Markov model-based adaptive CAC scheme to reduce the new call blocking probability. The scheme formulates the resource allocation problem as a Markov chain model. It considers calling request as RT and NRT, connectivity as NC and HC. The scheme uses the PRB allocation strategy by dynamically reserves resources for handoff calls based on traffic conditions and uses the remaining available resources to accept all types of calls. It degrades lower priority calls under heavy traffic or when the system is congested to accept more calls. Lower priority calls are always degraded when higher priority calls arrive, and there are no sufficient resources to admit them. The scheme decreases call blocking probability for higher priority classes and guarantees fair resource sharing among different traffic types. However, the scheme fails to utilize resources efficiently. It also starves lower priority calls due to the degradation strategy whenever a higher priority call arrives.

Authors in [28] proposed an efficient call admission control scheme to increase resource utilization and reduces call dropping probability of different user requests. The scheme classifies incoming requests into $\mathrm{HC}$ and $\mathrm{NC}$ and gives higher priority to $\mathrm{HC}$ without neglecting the NC. It uses a system priority approach for four service classes; NC-NGBR, HC-NGBR, NC-GBR, and HC-GBR. The scheme checks whether there are available PRBs in the network to admit either an NC or an HC. It accepts an NC if the number of requested PRBs is less than the available PRBs in the network; otherwise, the request is rejected.

Furthermore, the scheme accepts an HC request if the number of requested PRBs is less than the available PRBs and the reserved PRBs for HCs in the network; otherwise, the request is rejected. The scheme increases PRB utilization and also reduces call dropping probability for HCs. However, it wastes resources because, in a situation where there are no frequent arrivals of HCs, the reserved PRBs are wasted.

In [29], a blocking based call admission control scheme reduces the call dropping rate for users that requires handover frequently. The scheme takes into consideration some UE parameters such as call type, characteristics, channel quality, and available resources. It classifies incoming calls into two categories; handover calls and new calls. Each category is further separated into three types based on the QoS requirements, such as Real-time voice (RT voice), Real-time video (RT video), and Non-real time (NRT), given RT voice the highest priority. At the same time, NRT was given the lowest priority. The scheme checks the availability of resources by computing the occupation ratio, which is the ratio of the number of used resources to the total system resources. It admits an incoming call if the occupation ratio is less than twothirds; otherwise, if the occupation ratio is more than two-thirds, then the call is blocked. It no longer admits a call once the occupation ratio is equal to one. The scheme reduces call dropping probability for handover calls because they are given higher priority. However, NRT traffic, which has lower priority, experiences a higher blocking rate.

The authors [7] presented a call admission control scheme for real-time and non-real-time traffic to reduce the CBP and CDP of different users. The scheme is an improvement of nonprioritize schemes, which gave equal treatment to both $\mathrm{NC}$ and $\mathrm{HC}$. It classifies call requests into the new call (NC) and handoff call (HC) and then gives $\mathrm{HC}$ the highest priority while NC will be given the lowest priority. The scheme computes traffic intensity for both $\mathrm{HC}$ and $\mathrm{NC}$ and has a low or high threshold. It admits an HC request when HC's traffic intensity is less than or equal to the threshold value for $\mathrm{HC}$; otherwise, the call request is rejected.

Similarly, the scheme admits an NC request when NC's traffic intensity is less than the threshold value for $\mathrm{NC}$; otherwise, the request is rejected. The scheme reduces call dropping probability HC and also increases the overall system throughput. However, it increases CBP for NC due to the higher priority given to HC. The scheme only considers traffic intensity as an admission criterion without considering resources and other critical factors such as PRBs and other network conditions. 
A fuzzy-based decisive approach for call admission control is presented in [30] to reduce the handoff dropping probability and ensure QoS calls in the LTE network. The scheme extracts the channel and user parameters of a call request and then passes the fuzzy module parameters. The module computes the appropriate output and then decides on whether to accept or blocks a new call. The scheme admits a call if the fuzzy module's output is below the threshold set for new calls and then blocks a call request when the output is above the threshold set. If the QoS of an admitted call is compromised, the scheme allocates extra resources to it; otherwise, the call is dropped if there are no extra resources. The scheme increases the throughput and reduces the dropping probability of handoff calls. However, more new calls are blocked, and the QoS of new calls is not guaranteed because of the higher priority given to the scheme's handoff calls.

A study in [1] presented a QoS-aware call admission control (QA-CAC) scheme to guarantee the QoS and increase the throughput of real-time calls. A new call is accepted if the requested bandwidth is less than or equal to the available bandwidth; else, a degradation mechanism is applied on admitted non-real-time calls. The mechanism degrades all admitted non-real-time calls and then adds the degraded bandwidth to the available bandwidth to admit the requested calls. If the available bandwidth is less than or equal to the requested bandwidth, the call is admitted; otherwise rejected. The QA-CAC guarantees the QoS of RT calls, thereby increasing the throughput of RT calls and also reduces the dropping rate of RT calls. However, the scheme wastes much bandwidth when the degraded bandwidth is less than the requested bandwidth. The scheme also reduces the throughput of NRT calls due to the degradation approach applied to NRT calls.

Table 1 shows a summary of the CAC schemes that have been reviewed for this work. The name, strength(s), and weakness(s) of each scheme are mentioned.

Table 1. Summary of CAC schemes reviewed

\begin{tabular}{|c|c|c|c|}
\hline $\mathbf{S} / \mathbf{N}$ & Name of Scheme & Strength(s) & Weakness(s) \\
\hline 1 & $\begin{array}{l}\text { A novel Radio admission control } \\
\text { scheme for multiclass services } \\
{[13]}\end{array}$ & $\begin{array}{l}\text { The scheme reduces the CBP of users } \\
\text { and also gurantee the QoS of some } \\
\text { service types. }\end{array}$ & $\begin{array}{l}\text { - QoS of lower pririty users due } \\
\text { to the fact that they are } \\
\text { degraded whenever } \\
\text { resources are not suffiient. } \\
\text { - It also increases in the CDP of } \\
\text { these users. }\end{array}$ \\
\hline 2 & $\begin{array}{l}\text { A resource-estimated } \mathrm{CAC} \\
\text { scheme [14] }\end{array}$ & $\begin{array}{l}\text { It Improves QoS and increases } \\
\text { resource utilization. }\end{array}$ & $\begin{array}{l}\text { It Increases handoff dropping } \\
\text { probability. }\end{array}$ \\
\hline 3 & $\begin{array}{l}\text { Delay Aware Call Admission } \\
\text { Control (DACAC) scheme [15] }\end{array}$ & $\begin{array}{l}\text { It guarantees QoS for different service } \\
\text { type. }\end{array}$ & $\begin{array}{l}\text { It increases new call blocking } \\
\text { probability. }\end{array}$ \\
\hline 4 & $\begin{array}{l}\text { Downlink CAC with look-ahead } \\
\text { calls [16] }\end{array}$ & $\begin{array}{l}\text { It utilizes resources efficiently when } \\
\text { traffic is low. }\end{array}$ & $\begin{array}{l}\text { - Resources are underutilized } \\
\text { when the traffic is high. } \\
\text { - It increases blocking } \\
\text { probability for new immediate } \\
\text { calls and handoff calls. }\end{array}$ \\
\hline 5 & $\begin{array}{l}\text { Two-stage call admission } \\
\text { control scheme [17] }\end{array}$ & $\begin{array}{l}\text { It reduces call blocking and dropping } \\
\text { probability for GBR requests, } \\
\text { guaranteed QoS of admitted calls and } \\
\text { increase the overall system capacity. }\end{array}$ & $\begin{array}{l}\text { - It increases call blocking and call } \\
\text { dropping probability of NGBR } \\
\text { requests. }\end{array}$ \\
\hline 6 & $\begin{array}{l}\text { Adaptive call admission control } \\
\text { scheme [11] }\end{array}$ & $\begin{array}{l}\text { The scheme reduces the handoff } \\
\text { dropping probability of handoff calls } \\
\text { because they are given higher priority. }\end{array}$ & $\begin{array}{l}\text { Increase the call blocking } \\
\text { probability of new call requests. } \\
\text { It also reduces the utilization of } \\
\text { network resource because of the } \\
\text { reservation strategy employed } \\
\text { by the scheme. }\end{array}$ \\
\hline 7 & $\begin{array}{l}\text { Utility Based Scheduling and } \\
\text { Call Admission Control } \\
\text { (UBSCAC) scheme [18] }\end{array}$ & It reduces CDP for HC. & $\begin{array}{l}\text { - It increases CBP for NC } \\
\text { - Users with bad channels are } \\
\text { starved due to degradation. }\end{array}$ \\
\hline 8 & $\begin{array}{l}\text { Hybrid Adaptive Call Admission } \\
\text { Control (HCAC) scheme [19] }\end{array}$ & $\begin{array}{l}\text { It reduces } \\
\text { probability. }\end{array}$ & $\begin{array}{l}\text { It increases new call blocking } \\
\text { probability under heavy traffic. }\end{array}$ \\
\hline
\end{tabular}


9 Efficient channel state based call admission control for nonreal time traffic in LTE (3GPP) networks [20]

10 Call Admission Control Statisfying Delay Constraint for Machine-to-Machine communications Advanced [21]

11 An admission control scheme - It reduces CBP and CDP for voice calls. for video telephony services in wireless networks [22]

12 QoS based call admission control and resource allocation scheme for LTE femtocell networks [22]

13 Call admission control scheme for LTE femtocell networks [24] control for LTE systems (LTE- higher priority calls FIAC) [25]

- It guarantees fair among service types.

15 A fuzzy approach for call - It reduces call blocking and dropping admission control [26] probability for data services and ensures QoS provisioning for both new and handoff calls of data services

16 Markov model-based adaptive - It decreases call blocking probability CAC scheme [27] for higher priority class

- It guarantees fair resource sharing among different traffic types.
17 An efficient call admission - It increases PRB utilization. control scheme [28] - It reduces call dropping probability for HCs.

18 Blocking-based call admission control scheme [29]

19 Call admission control scheme for real time and non-real time traffic [7]

20 Fuzzy-based decisive approach The scheme increases the throughput for call admission control in LTE networks [30]

21 QoS-Aware Call $\begin{aligned} & \text { admission } \\ & \text { control scheme for LTE } \\ & \text { networks [1] }\end{aligned}$
and reduces the dropping probability of handoff calls.

The scheme reduces call dropping probability for handover calls.

The scheme reduces call dropping probability HC.
It increases the CBP and CDP of calls with bad channel condition.

It increases call blocking probability of calls.

It increases CBP and CDP for video calls

QoS of lower priority calls i.e. data calls is not maintained because they are given lower priority.

It increases CDP when the queued requests reaches queue timeout.

It increases call blocking and call dropping probability for lower priority calls.

It increases CBP and CDP of higher priority calls.

Lower priority calls are not treated fairly due to the degradation strategy applied on them whenever a higher priority call arrives. It wastes resources in a situation where there is no frequent arrivals of HCs, the reserved PRBs are wasted. NRT traffic which has lower priority experiences higher blocking rate.

It increases CBP for $\mathrm{NC}$ due to the higher priority given to $\mathrm{HC}$.

More new calls are blocked and the QoS of new calls is not guaranteed because of the higher priority given to the hand off calls by the scheme.

The scheme wastes a lot of bandwidth when the degraded bandwidth is less than the requested bandwidth and also reduces the throughput of NRT calls as a result of the degradation approach applied to NRT calls.

\section{Conclusion}

This paper presented a highlight on the 3GPP network by explaining the LTE network's concept, aim, and architecture. It further gave an overview of one of the RRM techniques known as CAC by defining it, explaining how it operates, and stating its importance in any network. It 
explains how the traditional CAC scheme operates. The paper further review some of the existing CAC schemes that were proposed for LTE networks. The operation, strength, and weakness of each of the schemes were highlighted. Based on reviews of these schemes, each scheme was developed for a particular purpose. Some of the schemes were developed to guarantee the QoS of existing and new calls; some were proposed to utilize available network resources. Other schemes were proposed for reducing of CBP and CDP of calls. This paper is presented as a stepping stone for researchers who want to extents their research in call admission control in LTE.

\section{References}

[1] M. M. Umar, A. Mohammed, A. Roko, A. Y. Tambuwal, and A. Abdulazeez, "QoS-aware call admission control (QA-CAC) scheme for LTE networks.," in 2019 15th International Conference on Electronics, Computer and Computation (ICECCO), Dec. 2019, pp. 1-5, doi: 10.1109/ICECCO48375.2019.9043228.

[2] S. Orduen Yese, A. Abdulazeez, A. Mohammed, M. Malami Umar, and Z. Yusuf Yeldu, "A Survey on Call Admission Control Schemes in LTE," Int. J. Comput. Sci. Eng. Surv., vol. 10, no. 5, pp. 1-20, Oct. 2019, doi: 10.5121/ijcses.2019.10501.

[3] A. Kumar, A. Aswal, and L. Singh, "4G wireless technology: a brief review," Int. J. Eng. Manag. Res., vol. 3, no. 2, pp. 35-43, 2013. Available: Google Scholar.

[4] ETSI, "Evolved Universal Terrestrial Radio Access (E-UTRA) and Evolved Universal Terrestrial Radio Access Network (E-UTRAN) (Release 10) - Overall description stage 2, 3rd Generation Partnership Project (3GPP), TS 36.300, v.10.2.0. 2010," 2010. Available: https://www.etsi.org/deliver/etsi_ts/136300_136399/136300/10.02.00_60/ts_136300v100200 p.pdf.

[5] D. Kakadia, J. Yang, and A. Gilgur, "Evolved Universal Terrestrial Radio Access Network (EUTRAN)," in Network Performance and Fault Analytics for LTE Wireless Service Providers, New Delhi: Springer, 2017, pp. 61-81, doi: 10.1007/978-81-322-3721-1.

[6] Navita and Amandeep, "Performance analysis of OFDMA, MIMO and SC-FDMA technology in 4G LTE networks," in 2016 6th International Conference - Cloud System and Big Data Engineering (Confluence), Jan. 2016, pp. 554-558, doi: 10.1109/CONFLUENCE.2016.7508181.

[7] M. Maharazu, Z. M. Hanapi, A. Abdullah, and A. Muhammed, "Call admission control for real-time and non-real-time traffic for vehicular LTE downlink networks," in Kim K., Joukov N. (eds) Mobile and Wireless Technologies 2017. ICMWT 2017. Lecture Notes in Electrical Engineering, vol 425, Singapore: Springer Singapore, 2018, pp. 46-53, doi: 10.1007/978-981-10-5281-1_6.

[8] M. H. Ahmed, "Call admission control in wireless networks: a comprehensive survey," IEEE Commun. Surv. Tutorials, vol. 7, no. 1, pp. 49-68, 2005, doi: 10.1109/COMST.2005.1423334.

[9] R. Kwan, R. Arnott, R. Trivisonno, and M. Kubota, "On pre-emption and congestion control for LTE systems," in 2010 IEEE 72nd Vehicular Technology Conference - Fall, Sep. 2010, pp. 1-5, doi: 10.1109/VETECF.2010.5594476.

[10] S. A. AlQahtani, "Users' classification-based call admission control with adaptive resource reservation for LTE-A networks," J. King Saud Univ. - Comput. Inf. Sci., vol. 29, no. 1, pp. 103-115, Jan. 2017, doi: 10.1016/j.jksuci.2015.12.002.

[11] F. Zarai, K. Ben Ali, M. S. Obaidat, and L. Kamoun, "Adaptive call admission control in 3GPP LTE networks," Int. J. Commun. Syst., vol. 27, no. 10, pp. 1522-1534, Oct. 2014, doi: 10.1002/dac.2415.

[12] A. A. Shaikh, B. S. Chowdhry, A. K. Baloch, and A. H. Pathan, "Radio resource management strategies in 3G UMTS network," in National Conference on Emerging Technologies 2004, 2004, pp. 48-51. Available: citeseerx.ist.psu.edu/viewdoc/download?doi=10.1.1.123.6479\&rep=rep1\&type=pdf.

[13] M. Qian, Y. Huang, J. Shi, Y. Yuan, L. Tian, and E. Dutkiewicz, "A novel radio admission control scheme for multiclass services in LTE systems," in GLOBECOM 2009 - 2009 IEEE Global Telecommunications Conference, Nov. 2009, pp. 1-6, doi: 10.1109/GLOCOM.2009.5426190. 
[14] S. J. Bae, J. J. Lee, B.-G. Choi, S. Kwon, and M. Y. Chung, "A resource-estimated call admission control algorithm in 3GPP LTE system," in Gervasi O., Taniar D., Murgante B., Laganà A., Mun Y., Gavrilova M.L. (eds) Computational Science and Its Applications - ICCSA 2009. ICCSA 2009. Lecture Notes in Computer Science, vol 5593, Berlin, Heidelberg: Springer-Verlag Berlin Heidelberg, 2009, pp. 250 260, doi: 10.1007/978-3-642-02457-3_22.

[15] S. J. Bae, B.-G. Choi, M. Y. Chung, J. J. Lee, and S. Kwon, "Delay-aware call admission control algorithm in 3GPP LTE system," in TENCON 2009 - 2009 IEEE Region 10 Conference, Nov. 2009, pp. 1-6, doi: 10.1109/TENCON.2009.5396157.

[16] F. M. Sallabi and K. Shuaib, "Downlink call admission control algorithm with look-ahead calls for 3GPP LTE mobile networks," in Proceedings of the 2009 International Conference on Wireless Communications and Mobile Computing Connecting the World Wirelessly - IWCMC '09, 2009, pp. 712715, doi: 10.1145/1582379.1582534.

[17] C. Kosta, T. Sodunke, M. Shateri, and R. Tafazolli, "Two-stage call admission control policy for LTE systems," in Proceedings of the 6th International Wireless Communications and Mobile Computing Conference on ZZZ - IWCMC '10, 2010, pp. 1101-1105, doi: 10.1145/1815396.1815648.

[18] J. Vijay Franklin and K. Paramasivam, "Utility based scheduling and call admission control for LTE (3GPP) networks," J. Inf. Technol. Softw. Eng., vol. 02, no. 05, 2012, doi: 10.4172/21657866.1000113.

[19] J. Vijay Franklin and K. Paramasivam, "Hybrid adaptive call admission control mechanism for ensuring QoS in 3GPP: LTE networks," Int. J. Comput. Appl., vol. 42, no. 21, pp. 36-41, Mar. 2012, doi: $10.5120 / 5838-8162$.

[20] J. Vijay Franklin and K. Paramasivam, "Efficient channel state based call admission control for non real time traffic in LTE (3GPP) networks," Int. J. Comput. Sci. Issues, vol. 2, no. 2, pp. 231-237, 2012, [Online]. Available: https://core.ac.uk/download/pdf/25718569.pdf.

[21] K. Jun, "Call Admission Control Satisfying Delay Constraint for Machine-to-Machine Communications in LTE-Advanced," in Kim T., Ko D., Vasilakos T., Stoica A., Abawajy J. (eds) Computer Applications for Communication, Networking, and Digital Contents. FGCN 2012. Communications in Computer and Information Science, vol 350, Berlin, Heidelberg: Springer-Verlag Berlin Heidelberg, 2012, pp. 48-55, doi: 10.1007/978-3-642-35594-3_7.

[22] I. A. Gudkova and K. E. Samouylov, "Modelling a Radio Admission Control Scheme for Video Telephony Service in Wireless Networks," in Andreev S., Balandin S., Koucheryavy Y. (eds) Internet of Things, Smart Spaces, and Next Generation Networking. ruSMART 2012, NEW2AN 2012. Lecture Notes in Computer Science, vol 7469, Berlin, Heidelberg: Springer-Verlag Berlin Heidelberg, 2012, pp. 208-215, doi: 10.1007/978-3-642-32686-8_19.

[23] C. Olariu, J. Fitzpatrick, P. Perry, and L. Murphy, "A QoS based call admission control and resource allocation mechanism for LTE femtocell deployment," in 2012 IEEE Consumer Communications and Networking Conference (CCNC), Jan. 2012, pp. 884-888, doi: 10.1109/CCNC.2012.6181184.

[24] J. Wang and Y. Qiu, "A new call admission control strategyfor LTE femtocell networks," in Proceedings of the 2nd International Conference on Advances in Computer Science and Engineering, 2013, pp. 334-338, doi: 10.2991/cse.2013.75.

[25] F. Furqan, D. B. Hoang, and I. B. Collings, "LTE-Advanced fair intelligent admission control LTEFIAC," in Proceeding of IEEE International Symposium on a World of Wireless, Mobile and Multimedia Networks 2014, Jun. 2014, pp. 1-4, doi: 10.1109/WoWMoM.2014.6919015.

[26] C. B. T. Ovengalt, K. Djouani, and A. Kurien, "'A fuzzy approach for call admission control in LTE networks,'” Procedia Comput. Sci., vol. 32, pp. 237-244, 2014, doi: 10.1016/j.procs.2014.05.420.

[27] K. Ben Ali, M. S. Obaidat, F. Zarai, and L. Kamoun, "Markov model-based adaptive CAC scheme for 3GPP LTE femtocell networks," in 2015 IEEE International Conference on Communications (ICC), Jun. 2015, pp. 6924-6928, doi: 10.1109/ICC.2015.7249429.

[28] R. Khdhir, K. Mnif, A. Belghith, and L. Kamoun, “An efficient call admission control scheme for LTE and LTE-A networks," in 2016 International Symposium on Networks, Computers and Communications (ISNCC), May 2016, pp. 1-6, doi: 10.1109/ISNCC.2016.7746068. 
[29] S. Namwanta and N. Ruangchaijatupon, "Blocking rate based call admission control for call dropping mitigation and QoS based prioritization," in 2017 International Electrical Engineering Congress (iEECON), Mar. 2017, pp. 1-4, doi: 10.1109/IEECON.2017.8075852.

[30] V. S. Jadhav and U. D. Kolekar, "Fuzzy-based decisive approach for call admission control in the LTE networks," Evol. Intell., Aug. 2019, doi: 10.1007/s12065-019-00270-1. 